\title{
ON SOME SPACES OF VECTOR-VALUED BOUNDED FUNCTIONS
}

\author{
J.C. FERRANDO
}

\begin{abstract}
Assuming $\Sigma$ is an algebra of subsets of a non-empty set $\Omega$ and $X$ is a normed space, I investigate whether or not certain barrelledness conditions, some of them introduced in the seventies by Saxon and Valdivia, are enjoyed by several subspaces of the linear space of all those bounded $X$-valued functions defined on $\Omega$ which are the uniform limit of a sequence of $X$-valued $\Sigma$-simple functions equipped with the supremum-norm.
\end{abstract}

\section{Preliminaries}

In what follows $\Omega$ will be a non-empty set, $\Sigma$ an algebra of subsets of $\Omega$ and $X$ a normed space. By $\ell_{0}^{\infty}(\Sigma, X)$ we denote the linear space over the field $\mathrm{K}$ of the real or complex numbers of all $X$-valued $\Sigma$-simple functions defined on $\Omega$. If $X=\mathrm{K}$, we will write $\ell_{0}^{\infty}(\Sigma)$ instead of $\ell_{0}^{\infty}(\Sigma, \mathrm{K})$. We represent by $B(\Sigma, X)$, or by $B(\Omega, X)$, the linear space over $\mathrm{K}$ of all bounded $X$-valued functions defined on $\Omega$ which are the uniform limit of a sequence in $\ell_{0}^{\infty}(\Sigma, X)$. We will assume that $B(\Sigma, X)$ is equipped with the supremum-norm $\|f\|=\sup \{\|f(\omega)\|: \omega \in \Omega\}$ and will consider $\ell_{0}^{\infty}(\Sigma, X)$ as a subspace of $B(\Sigma, X)$. If $\Sigma$ is not a $\sigma$-algebra, then $f^{-1}(x)$, for $f \in B(\Sigma, X)$ and $x \in X$, need not be an element of $\Sigma$, although certainly it is an element of the $\sigma$ algebra generated by $\Sigma$. The subspace of $B(\Sigma, X)$ formed by all countablyvalued functions will be denoted by $K(\Sigma, X)$ or by $K(\Omega, X)$, while $K_{0}(\Sigma, X)$ will stand for the linear subspace of $K(\Sigma, X)$ consisting of all those countably-valued $f$ for which there exists a countable partition $\left\{A_{n}, n \in \mathrm{N}\right\}$ of $\Omega$ by elements of $\Sigma$ such that $f$ is constant in each set $A_{n}, n \in \mathrm{N}$. If $f \in K(\Sigma, X)$ is such that $f^{-1}(x) \in \Sigma$ for all $x \in X$, then clearly $f \in K_{0}(\Sigma, X)$. Naturally $K_{0}(\Sigma, X)$ coincides with $K(\Sigma, X)$ whenever $\Sigma$ is a $\sigma$-algebra, although in general $K_{0}(\Sigma, X)$ is a dense subspace of $K(\Sigma, X)$, since $\ell_{0}^{\infty}(\Sigma, X) \subseteq K_{0}(\Sigma, X)$. When $X=\mathrm{K}$ we will write $K_{0}(\Sigma)$ instead of $K_{0}(\Sigma, \mathrm{K})$. If $E \subseteq \Omega$ we will represent by $B(E, X)$ the subspace of $B(\Sigma, X)$ of all those functions $f$ whose

This paper has been supported by DGICYT grant PB94-0535

Received July 24, 1996. 
support is contained in $E$. Note that the restriction $\Sigma_{\mid E}:=\{Y \cap E: Y \in \Sigma\}$ is an algebra on $E$, and if $E \in \Sigma$ then $B(E, X)$ is isometric to the space $B\left(\Sigma_{\mid E}, X\right)$. Finally, we will denote by $\operatorname{St}(I, X)$ the linear space of the $X$-valued step functions defined in the semiclosed unit interval $I=(0,1]$ provided with the supremum-norm, and by $\operatorname{Reg}(I, X)$ the $X$-valued regulated function space. Assuming that $\Omega$ coincides with $I$ and $\Sigma$ is the algebra of finite unions of right-semiclosed intervals contained in $I$, we may identify both spaces with their corresponding subspaces of $B(\Sigma, X)$. The reader is referred to Section 5 for a detailed definition of these spaces.

Let us remark that if $\omega \in \Omega$, the mapping $f \rightarrow f(\omega)$ from $B(\Sigma, X)$ onto $X$ is a quotient, so $X$ shares the same barrelledness property as $B(\Sigma, X)$ does for those barrelledness conditions considered in this paper.

A Hausdorff locally convex space (1.c.s. in brief) $E$ is said to be barrelled of class 1 or suprabarrelled [13] if, given an increasing sequence $\left(E_{n}\right)$ of subspaces of $E$ covering $E$, there is some $E_{m}$ which is dense and barrelled. A 1.c.s. $E$ is said to be barrelled of class $n, n>1$, [5] if, given an increasing sequence $\left(E_{n}\right)$ of subspaces of $E$ covering $E$, there is some $E_{m}$ which is dense and barrelled of class $n-1$. If $E$ is barrelled of class $n$ for each $n \in \mathrm{N}, E$ is called barrelled of class $\aleph_{0}$ [5]. A 1.c.s. $E$ is said to be totally barrelled [15] (TB for short) if, given a sequence $\left(E_{n}\right)$ of subspaces of $E$ covering $E$, there is one of them which is barrelled and its closure is of finite codimension in $E$. If $E$ is metrizable, this is equivalent (see 9.3.3 of [10]) to saying that there is some $E_{m}$ which is barrelled. A 1.c.s. $E$ is said to be Baire-hyperplane [14] (BH for short) if it cannot be covered by any sequence of closed hyperplanes. A 1.c.s. $E$ is said to be unordered Baire-like (UBL in brief) [12] if, given a sequence of closed absolutely convex subsets of $E$ covering $E$, one of them is a neighbourhood of the origin in $E$. A 1.c.s. $E$ is said to be Baire-like (BL in brief) [11] if, given an increasing sequence of closed absolutely convex subsets of $E$ covering $E$, then one of them is a neighbourhood of the origin in $E$. One has that

$$
\begin{aligned}
\mathrm{UBL} & \Rightarrow \mathrm{BH} \\
\mathrm{UBL} & \Rightarrow \mathrm{TB} \Rightarrow \text { barrelled space of class } \aleph_{0} \Rightarrow \mathrm{BL} \Rightarrow \text { barrelled }
\end{aligned}
$$

While, as is well-known, $\ell_{0}^{\infty}(\Sigma, X)$ is barrelled if and only if $\ell_{0}^{\infty}(\Sigma)$ is barrelled and $X$ is finite-dimensional [7], we have that $B(\Sigma, X)$ is barrelled if and only if $X$ is barrelled [9], and - assuming that $\Sigma$ is a $\sigma$-algebra $-B(\Sigma, X)$ is barrelled of class $n$ [of class $\aleph_{0}$ ], with $n \geq 1$, if and only if $X$ is barrelled of class $n$ [resp. of class $\aleph_{0}$ ] [6] (see also [5]). In Problem 11.16 of [5] we ask whether or not $B(\Sigma, X)$ is UBL whenever $X$ is UBL. In this paper we show that $B(\Sigma, X)$ is $\mathrm{BH}$ if and only if $X$ is $\mathrm{BH}$ and, using some ideas from [3], we 
give some conditions for $B(\Sigma, X)$ to be UBL. We also obtain several barrelledness conditions concerning the spaces $K_{0}(\Sigma, X)$ and $K(\Sigma, X)$ depending on the kind of barrelledness conditions exhibited by the range space $X$. Besides, we show that $\operatorname{St}(I, X)$ is never barrelled and that $\operatorname{Reg}(I, X)$ is barrelled if and only if $X$ is barrelled.

For a glance of the present research concerning barrelledness properties of spaces of vector-valued bounded [measurable] functions see [8], [1] and [4] [resp.[3]].

\section{The space $B(\Sigma, X)$}

THeORem 2.1. $B(\Sigma, X)$ is a $\mathrm{BH}$ space if and only if $X$ is a $\mathrm{BH}$ space.

Proof. If $B(\Sigma, X)$ is $\mathrm{BH}$, then obviously $X$ is $\mathrm{BH}$. Let us show that if $B(\Sigma, X)$ is not $\mathrm{BH}$, then $X$ is not $\mathrm{BH}$. Assume that $\left\{H_{n}: n \in \mathrm{N}\right\}$ is a sequence of closed hyperplanes of $B(\Sigma, X)$ covering $B(\Sigma, X)$ and define the following closed linear subspaces of $X$

$$
L_{n}=\left\{x \in X: \chi_{A} x \in H_{n} \text { for each } A \in \Sigma\right\}
$$

for each $n \in \mathrm{N}$. Clearly $\ell_{0}^{\infty}(\Sigma, X) \not \subset H_{n}$, since otherwise $H_{n}=B(\Sigma, X)$, a contradiction. Hence there is some $f_{n} \in \ell_{0}^{\infty}(\Sigma, X)-H_{n}$ such that $B(\Sigma, X)=$ $\left\langle f_{n}\right\rangle \oplus H_{n}$. Assuming that $f_{n}=\sum_{i=1}^{m} z_{n i} \chi_{E_{n i}}$ where $E_{n i} \in \Sigma, \quad E_{n i} \neq \emptyset$ for $1 \leq i \leq m$, and $E_{n i} \cap E_{n j}=\emptyset$ if $i \neq j$, there must be some positive integer $k$ with $1 \leq k \leq m$ such that $\chi_{E_{n k}} z_{n k} \notin H_{n}$. This implies that $z_{n k} \notin L_{n}$. Consequently, for each $n \in \mathrm{N}$ there is a closed hyperplane $X_{n}$ in $X$ such that $L_{n} \subseteq X_{n}$. So, it suffices to show that $\bigcup_{i=1}^{\infty} L_{i}=X$. Now given $z \in X, z \neq 0$, then $L(z):=\left\{f(\cdot) z: f \in \ell_{\infty}(\Sigma)\right\}$, where $\ell_{\infty}(\Sigma)$ denotes the completion of the scalar-valued $\Sigma$-simple function space $\ell_{0}^{\infty}(\Sigma)$, is a subspace of $B(\Sigma, X)$ isomorphic to the Banach space $\ell_{\infty}(\Sigma)$. Hence, according to the Baire category theorem, there is some $H_{j}$ such that $L(z) \subseteq H_{j}$. Particularly $\chi_{A} z \in H_{j}$ for each $A \in \Sigma$, which implies that $z \in L_{j}$.

Lemma 2.2. Let $\left\{W_{n}: n \in \mathrm{N}\right\}$ be a sequence of closed absolutely convex subsets of $B(\Sigma, X)$ covering $B(\Sigma, X)$. If $X$ is $\mathrm{UBL}$ there exists some $i \in \mathrm{N}$ such that $W_{i}$ absorbs the set $\left\{\chi_{A} x: A \in \Sigma,\|x\| \leq 1\right\}$.

Proof. Let us define the following closed absolutely convex subsets of $X$

$$
V_{n m}=\left\{x \in X: \chi_{A} x \in m W_{n} \text { for each } A \in \Sigma\right\}
$$

for each $n, m \in \mathrm{N}$. Given $z \in X, \quad z \neq 0$, then as before $L(z):=$ $\left\{f(\cdot) z: f \in \ell_{\infty}(\Sigma)\right\}$ is a subspace of $B(\Sigma, X)$ isomorphic to the Banach space $\ell_{\infty}(\Sigma)$. Consequently there exist some $r, s \in \mathrm{N}$ such that $\chi_{A} z \in s W_{r}$ for each $A \in \Sigma$. This implies that $z \in V_{r s}$, and therefore we have 
$\cup\left\{V_{n m}: n, m \in \mathrm{N}\right\}=X$. As $X$ is $\mathrm{UBL}$ there are $i, j, k \in \mathrm{N}$ such that $k V_{i j}$ contains the closed unit ball of $X$. Hence $\chi_{A} x \in j k W_{i}$ for each $x \in X$ with $\|x\| \leq 1$ and each $A \in \Sigma$. Thus $W_{i}$ absorbs the set $\left\{\chi_{A} x: A \in \Sigma,\|x\| \leq 1\right\}$.

Theorem 2.3. Assuming that $X$ is a UBL space, then $B(\Sigma, X)$ is UBL if and only if it is TB.

Proof. Clearly, if $B(\Sigma, X)$ is UBL, then it is TB. Assume conversely that $B(\Sigma, X)$ is TB and let $\left\{W_{n}: n \in \mathrm{N}\right\}$ be a sequence of closed absolutely convex subsets of $B(\Sigma, X)$ covering $B(\Sigma, X)$. It suffices to notice that, according to the previous lemma, there is an $i \in \mathrm{N}$ such that $\ell_{0}^{\infty}(\Sigma, X) \subseteq\left\langle W_{i}\right\rangle$, and since $\ell_{0}^{\infty}(\Sigma, X)$ is dense in $B(\Sigma, X)$, this implies that $\left\langle W_{i}\right\rangle$ is dense in $B(\Sigma, X)$. Consequently, by means of Theorem 4.1 of [12], we may assume that $\left\{\left\langle W_{i}\right\rangle: i \in \mathbf{N}\right\}$ covers $B(\Sigma, X)$ and each $\left\langle W_{i}\right\rangle$ is dense in $B(\Sigma, X)$. But as $B(\Sigma, X)$ is TB, there must be some $j \in \mathrm{N}$ such that $\left\langle W_{j}\right\rangle$ is barrelled which, as can be easily proven, implies that $\left\langle W_{j}\right\rangle$ must be closed too. So $\left\langle W_{j}\right\rangle=B(\Sigma, X)$ and therefore, $B(\Sigma, X)$ being barrelled, $W_{j}$ is a neighbourhood of the origin in $B(\Sigma, X)$.

Lemma 2.4. Let $\left\{A_{n}: n \in \mathrm{N}\right\}$ be a sequence of pairwise disjoint subsets of $\Omega$. If $\left(T_{n}\right)$ is a sequence of closed absolutely convex subsets of $B(\Sigma, X)$ covering $B(\Sigma, X)$, there are $m \in \mathrm{N}$ and $J_{m} \subseteq \mathrm{N}$, with $J_{m}$ finite or empty, such that $T_{m}$ absorbs the closed unit ball of the subspace $B\left(\cup\left\{A_{n}: n \notin J_{m}\right\}, X\right)$.

Proof. Assume the lemma is not true and denote by $\left\{Q_{i}: i \in \mathrm{N}\right\}$ the countable family $\left\{m T_{n}: m, n \in \mathrm{N}\right\}$. For each $i \in \mathrm{N}$, let

$$
f_{i} \in B\left(\cup\left\{A_{n}: n \geq i\right\}, X\right)
$$

be such that $\left\|f_{i}\right\|=1$ and $f_{i} \notin Q_{i}$. Then, for each $\xi \in \ell_{1}$ the series $\sum_{i=1}^{\infty} \xi_{i} f_{i}$ converges in the completion $B(\Sigma, \widehat{X})$ of $B(\Sigma, X)$ to some $f_{\xi}$. But since $\sum_{i=1}^{\infty} \xi_{i} f_{i}(\omega)=0$ if $\omega \notin \bigcup_{n=1}^{\infty} A_{n}$ and $\sum_{i=1}^{\infty} \xi_{i} f_{i}(\omega)=\sum_{i=1}^{n} \xi_{i} f_{i}(\omega) \in X$ if $\omega \in A_{n}$, we must conclude that $f_{\xi} \in B(\Sigma, X)$. This implies that the linear mapping $\xi \rightarrow \sum_{i=1}^{\infty} \xi_{i} f_{i}$ from $\ell_{1}$ into $B(\Sigma, X)$ is well-defined (and continuous). Hence, the set $D=\left\{f_{\xi}: \xi \in \ell_{1}\right\}$ is a Banach disk in $B(\Sigma, X)$, and as $\left(T_{n}\right)$ covers $B(\Sigma, X)$, there must be some positive integer $j$ such that $T_{j}$ absorbs the set $D$. Consequently there is some $k \in \mathrm{N}$ such that $Q_{k} \supseteq D$, which leads to $f_{k} \in Q_{k}$, a contradiction.

Proposition 2.5 Let $\mathscr{A}$ be an algebra of subsets of $\mathrm{N}$ containing the finite sets. Then $B(\mathscr{A}, X)$ is UBL if and only if $X$ is UBL.

Proof. Suppose $X$ is UBL. Let $\left\{W_{n}: n \in \mathrm{N}\right\}$ be a sequence of closed absolutely convex subsets of $B(\mathscr{A}, X)$ covering $B(\mathscr{A}, X)$ and consider the partition $\{\{n\}: n \in \mathbf{N}\}$ of $\mathbf{N}$. According to Lemma 2.2 and Theorem 4.1 of 
[12] there is no loss of generality by assuming that $\ell_{0}^{\infty}(\mathscr{A}, X) \subseteq\left\langle W_{n}\right\rangle$ for each $n \in \mathrm{N}$. On the other hand, using Lemma 2.4, there are $m \in \mathrm{N}$ and $J_{m} \subseteq \mathrm{N}$, with $J_{m}$ finite or empty, such that $B\left(\mathrm{~N} \backslash J_{m}, X\right) \subseteq\left\langle W_{m}\right\rangle$. From these facts, and noticing that $B\left(J_{m}, X\right)=\ell_{0}^{\infty}\left(\mathscr{A}_{\mid J_{m}}, X\right)$, it follows that $B(\mathscr{A}, X)=\left\langle W_{m}\right\rangle$, so the conclusion is a consequence of the barrelledness of $B(\mathscr{A}, X)$.

\section{The space $K_{0}(\Sigma, X)$}

Lemma 3.1. Assume that $T$ is a barrel in the space $K_{0}(\Sigma, X)$. If $T$ absorbs the set $\left\{\chi_{A} x: A \in \Sigma,\|x\| \leq 1\right\}$, then $T$ is a neighbourhood of the origin in $K_{0}(\Sigma, X)$.

Proof. If $T$ is not a neighbourhood of the origin in $K_{0}(\Sigma, X)$, by density there is some $f_{1} \in \ell_{0}^{\infty}(\Sigma, X)$ with $\left\|f_{1}\right\|=1$ and $f_{1} \notin 2 T$. If $\left\{P_{1 m}: 1 \leq m \leq n_{1}\right\}$ is the (finite) partition of $\Omega$ induced by $f_{1}$, there exists a positive integer $k_{1}$, $1 \leq k_{1}<n_{1}$, such that $T$ does not absorb the unit ball of $\ell_{0}^{\infty}\left(P_{1 k_{1}}, X\right)$. Hence, setting $\Omega_{1}=P_{1 k_{1}}$ and $x_{1}$ being the constant value of $f_{1}$ in $\Omega_{1}$, there must be $f_{2} \in \ell_{0}^{\infty}\left(\Omega_{1}, X\right)$ with $\left\|f_{2}\right\|=1, f_{2} \notin 4 T$. If $\left\{P_{2 m}: 1 \leq m \leq n_{2}\right\}$ is the partition of $\Omega_{1}$ induced by $f_{2}$, there is some positive integer $k_{2}, 1 \leq k_{2} \leq n_{2}$, such that $T$ does not absorb the unit ball of $\ell_{0}^{\infty}\left(P_{2 k_{2}}, X\right)$. Set $\Omega_{2}=P_{2 k_{2}}$ and let $x_{2}$ be the constant value of $f_{2}$ in $\Omega_{2}$. Proceeding by recurrence we obtain a normalized sequence $\left(f_{n}\right)$ in $\ell_{0}^{\infty}(\Sigma, X)$, a sequence $\left(x_{n}\right)$ in $X$ with $\left\|x_{n}\right\| \leq 1$ for each $n \in \mathrm{N}$, and a decreasing sequence $\left(\Omega_{n}\right)$ of elements of the algebra $\Sigma$, such that $\Omega_{n} \subseteq \operatorname{supp} f_{n} \subseteq \Omega_{n-1}$, where $\Omega_{0}=\Omega$, and $f_{n} \notin 2 n T, f_{n}$ taking the constant value $x_{n}$ in $\Omega_{n}$, for each $n \in \mathrm{N}$. Setting $g_{n}=f_{n}-\chi_{\Omega_{n}} x_{n}$ for each $n$, as $T$ absorbs the set $\left\{\chi_{A} x: A \in \Sigma,\|x\| \leq 1\right\}$, there is some $k \in \mathrm{N}$ such that $\chi_{\Omega_{n}} x_{n} \in n T$ for each $n \geq k$. Hence $g_{n} \notin n T$ for each $n \geq k$. Now, due to the fact that the $g_{n}$ are disjointly supported, the subspace $\operatorname{span}\left\{g_{n}: n \geq k\right\}$ of $B(\Sigma, \widehat{X})$ is a copy of $c_{0}$ contained in $K_{0}(\Sigma, X)$. Hence there is some positive integer $j$ such that $g_{j} \in j T$, a contradiction.

Lemma 3.2. $K_{0}(\Sigma)$ is a barrelled space.

Proof. Assume the lemma is not true. Then there is a barrel $T$ in $K_{0}(\Sigma)$ which is not a neighbourhood in $K_{0}(\Sigma)$. As $T$ does not absorb the closed unit ball of $\ell_{0}^{\infty}(\Sigma)$ a standard argument (see for instance [2]) yields a pairwise disjoint sequence $\left(A_{n}\right)$ of elements of $\Sigma$ and a strictly increasing sequence $\left(n_{i}\right)$ of positive integers such that $\chi_{A_{i}} \notin n_{i} T$ for each $i \in \mathbf{N}$. So $\overline{\operatorname{span}}\left\{\chi_{A_{i}}: i \in \mathbf{N}\right\}$ is a copy of $c_{0}$ contained in $K_{0}(\Sigma)$ and therefore there must be some $k \in \mathrm{N}$ such that $\chi_{A_{k}} \in n_{k} T$, a contradiction.

THEOREM 3.3. $K_{0}(\Sigma, X)$ is barrelled if and only if $X$ is barrelled.

Proof. Suppose $X$ is barrelled. Let $T$ be a barrel in $K_{0}(\Sigma, X)$ and define 


$$
V_{m}=\left\{x \in X: \chi_{A} x \in m T \forall A \in \Sigma\right\}
$$

for each $m \in \mathrm{N}$. Then $\left\{V_{m}: m \in \mathrm{N}\right\}$ is an increasing sequence of closed absolutely convex subsets of $X$. If $z \in X, z \neq 0$, according to the previous lemma,

$$
L(z):=\left\{f(\cdot) z: f \in K_{0}(\Sigma)\right\}
$$

is a barrelled space contained in $K_{0}(\Sigma, X)$, and consequently there is some $k \in \mathrm{N}$, such that $\chi_{A} z \in k T$ for each $A \in \Sigma$, i.e., $z \in V_{k}$. Hence $\left\{V_{m}: m \in \mathrm{N}\right\}$ covers $X$. But $X$, being metrizable and barrelled, is a Baire-like space, which means that there exists some $j \in \mathrm{N}$ such that $V_{j}$ absorbs the closed unit ball of $X$. This implies that there is some $i \in \mathrm{N}$ such that $\chi_{A} x \in i j T$ for each $x \in X$ with $\|x\| \leq 1$ and each $A \in \Sigma$. Therefore $T$ absorbs the set $\left\{\chi_{A} x: A \in \Sigma,\|x\| \leq 1\right\}$ and the conclusion follows from Lemma 3.1.

Corollary 3.4. (Mendoza) $B(\Sigma, X)$ is barrelled if and only if $X$ is barrelled.

Proof. This is an obvious consequence of the previous theorem, since $K_{0}(\Sigma, X)$ is a dense subspace of $B(\Sigma, X)$.

Although $K_{0}(\Sigma, X)$ is always barrelled whenever $X$ is barrelled, this space need not be TB or BH even if $X$ is a Banach space, as the following example shows.

EXAmple 3.5. Assume that $\Omega=\mathrm{R}$ and let $\mathscr{M}$ be the algebra of all subsets $A$ of $\mathrm{R}$ such that either $A$ or $\mathrm{R} \backslash$ A contains a dense open set of the usual topology of $\mathrm{R}$. Let $\left(B_{n}\right)$ be the sequence of all open intervals whose extremes are both rational numbers. As we know, according to Lemma $3.2 K_{0}(\mathscr{M})$ is a barrelled space, and we are going to show that $K_{0}(\mathscr{M})$ is not $\mathrm{TB}$ or $\mathrm{BH}$.

Indeed, $L_{n}:=\left\{f \in K_{0}(\mathscr{M}): f\right.$ is constant in $\left.B_{n}\right\}$ is for each $n$ a closed linear subspace of $K_{0}(\mathscr{M})$ and the sequence $\left(L_{n}\right)$ covers $K_{0}(\mathscr{M})$, for given $f \in K_{0}(\mathscr{M})$, if $\left\{A_{n}: n \in \mathrm{N}\right\}$ is a partition of $\Omega$ by elements of $\mathscr{M}$ induced by $f$, since $\Omega$ is a Baire space there must be some $k$ such that $A_{k}$ contains a dense open set. So there exists a positive integer $m$ such that $B_{m} \subseteq A_{k}$, which shows that $f \in L_{m}$. As each $L_{n}$ is infinite-codimensional - if $\left(q_{i}\right)$ is an enumeration of the rational numbers contained in $B_{n}$, the set $\left\{\chi_{\left\{q_{i}\right\}}: i \in \mathrm{N}\right\}$ is linearly independent with respect to $L_{n}$ and $\chi_{\left\{q_{i}\right\}} \in K_{0}(\mathscr{M})$ for each $i$ since $\Omega \backslash\left\{q_{i}\right\}$ is a dense open set -, it follows that $K_{0}(\mathscr{M})$ is not a TB space. Finally, as $K_{0}(\mathscr{M})$ may be easily covered by a sequence $\left(H_{n}\right)$ of closed hyperplanes, then $K_{0}(\mathscr{M})$ is not a $\mathrm{BH}$ space. 


\section{The space $K(\Sigma, X)$}

For the next results $\Sigma$ will be a $\sigma$-algebra of subsets of $\Omega$. If $\pi$ is a countable partition of $\Omega$ by elements of $\Sigma$, we denote by $K(\pi, X)$ the linear subspace of $K(\Sigma, X)$ formed by all those functions $f$ such that $f(s)=f(t)$ for each $s, t \in A$ with $A \in \pi$, and represent by $\Pi$ the family of all countable partitions of $\Omega$ by elements of $\Sigma$.

Lemma 4.1. Let $\left\{A_{n}: n \in \mathrm{N}\right\}$ be a sequence of pairwise disjoint elements of $\Sigma$. If $V$ is an absolutely convex subset of $K(\Sigma, X)$ such that $V \cap K(\pi, X)$ is a neighbourhood of the origin in $K(\pi, X)$ for each $\pi \in \Pi$, then there is some $m \in \mathrm{N}$ such that $V$ absorbs the closed unit ball of $K\left(\cup\left\{A_{n}: n \geq m\right\}, X\right)$.

Proof. Otherwise there exists a normalized sequence $\left(f_{m}\right) \subseteq K(\Sigma, X)$ such that $f_{m} \in K\left(\cup\left\{A_{n}: n \geq m\right\}, X\right)$ and $f_{m} \notin m V$ for each $m \in \mathrm{N}$. Then $\sum_{m=1}^{\infty} \xi_{m} f_{m}$ converges in the Banach space $B(\Sigma, \widehat{X})$ to $f_{\xi}$ for $\xi=\left(\xi_{m}\right) \in \ell_{1}$, and reasoning as in Lemma 2.4 it is easy to note that $f_{\xi} \in K(\Sigma, X)$. As $f^{-1}(x) \in \Sigma$ for each $f \in B(\Sigma, X)$ and $x \in X$, then each $f \in K(\Sigma, X)$ induces some $\pi_{f} \in \Pi$ such that $f \in K\left(\pi_{f}, X\right)$. So meeting each $A_{n}$ respectively with the countable partitions induced by the supports of $f_{1}, \ldots, f_{n}$, it is not difficult to determine a $\pi \in \Pi$ independent of $\xi$ such that $f_{\xi} \in K(\pi, X)$. Therefore $\left\{f_{\xi}: \xi \in \ell_{1},\|\xi\| \leq 1\right\}$ is a Banach disk in $K(\pi, X)$ and hence there is some $k \in \mathbf{N}$ such that $f_{\xi} \in k V$ for each $\xi \in \ell_{1}$. In particular $f_{k} \in k V$, a contradiction.

Lemma 4.2. Let $V$ be an absolutely convex subset of $K(\Sigma, X)$ such that $V \cap K(\pi, X)$ is a neighbourhood of the origin in $K(\pi, X)$ for each $\pi \in \Pi$. If $\left\{\Omega_{n}: n \in \mathrm{N}\right\}$ is a decreasing sequence of elements of $\Sigma$ and $\left(x_{n}\right)$ is a bounded sequence in $X$, there is some positive integer $m$ such that $\chi_{\Omega_{n}} x_{n} \in m V$ for each $n \in \mathbf{N}$.

Proof. Set $A_{n}=\Omega_{n-1} \backslash \Omega_{n}$ for each $n \in \mathrm{N}$, where we write $\Omega_{0}=\Omega$, and let $\pi=\left\{A_{n}: n=0,1, \ldots\right\}$ where $A_{0}=\bigcap_{n=1}^{\infty} \Omega_{n}$. Since we have $\chi_{\Omega_{n}} x_{n}=$ $\chi_{A_{0}} x_{n}+\sum_{m=1}^{\infty} \chi_{A_{m}} y_{n m}$, with $y_{n m}=0$ for $1 \leq m \leq n$ and $y_{n m}=x_{n}$ for $m>n$, pointwise on $\Omega$, it follows that $\chi_{\Omega_{n}} x_{n} \in K(\pi, X)$ for each $n \in \mathrm{N}$. Choosing $M>0$ such that $\left\|x_{n}\right\| \leq M$ for each $n \in \mathrm{N}$ we have that $\left\|\chi_{\Omega_{n}} x_{n}\right\| \leq M$. Now, given that $V \cap K(\pi, X)$ is a neighbourhood of the origin in $K(\pi, X)$, there exists a positive integer $k$ such that $\chi_{\Omega_{n}} x_{n} \in k V$ for each $n \in \mathrm{N}$.

THEOREM 4.3. If $\Sigma$ is a $\sigma$-algebra, $K(\Sigma, X)$ is the locally convex hull of the family $\{K(\pi, X): \pi \in \Pi\}$

Proof. As each $f \in K(\Sigma, X)$ induces a partition $\pi_{f} \in \Pi$ such that $f \in K\left(\pi_{f}, X\right)$, then $K(\Sigma, X)=\bigcup\{K(\pi, X): \pi \in \Pi\}$. 
Let $V$ be an absolutely convex subset of $K(\Sigma, X)$ such that $V \cap K(\pi, X)$ is a neighbourhood of the origin in $K(\pi, X)$ for each $\pi \in \Pi$. We are going to prove that $V$ is a neighbourhood of the origin in $K(\Sigma, X)$.

Assume that $V$ is not a neighbourhood of the origin in $K(\Sigma, X)$. Then using Lemmas 4.1 and 4.2 we are going to obtain a bounded sequence $\left(g_{n}\right) \subseteq K(\Sigma, X)$ such that $g_{n} \notin n V$ for each $n \in \mathrm{N}$ and supp $g_{i} \cap \operatorname{supp} g_{j}=\emptyset$ if $i \neq j$. We outline the way to do this. Choose some $f_{1} \in K(\Omega, X)$ with $\left\|f_{1}\right\|=1$ such that $f_{1} \notin 2 V$. If $\left\{P_{1 m}: m \in \mathrm{N}\right\}$ is the partition of $\Omega$ by elements of $\Sigma$ induced by $f_{1}$, then according to Lemma 4.1 there is some positive integer $n_{1}$ such that $V$ absorbs the unit ball of $K\left(\cup\left\{P_{1 n}: n \geq n_{1}\right\}, X\right)$. Hence there exists a $1 \leq k_{1}<n_{1}$ such that $V$ does not absorb the unit ball of $K\left(P_{1 k_{1}}, X\right)$. Set $\Omega_{1}=P_{1 k_{1}}$ and let $x_{1}$ be the constant value of $f_{1}$ in $\Omega_{1}$. Again there must exist an $f_{2} \in K\left(\Omega_{1}, X\right)$ with $\left\|f_{2}\right\|=1$ and $f_{2} \notin 4 V$. Then, let $\left\{P_{2 m}: m \in \mathrm{N}\right\}$ be the partition of $\Omega_{1}$ by elements of $\Sigma$ induced by $f_{2}$. Using again Lemma 4.1 we obtain some positive integer $k_{2}$ such that $V$ does not absorb the unit ball of $K\left(P_{2 k_{2}}, X\right)$. Set $\Omega_{2}=P_{2 k_{2}}$ and let $x_{2}$ be the constant value of $f_{2}$ in $\Omega_{2}$. Proceeding by recurrence we obtain a normalized sequence $\left(f_{n}\right)$ in $K(\Sigma, X)$, a sequence $\left(x_{n}\right)$ in $X$ with $\left\|x_{n}\right\| \leq 1$ for each $n \in \mathrm{N}$ and a decreasing sequence $\left(\Omega_{n}\right)$ of elements of $\Sigma$, such that $\Omega_{n} \subseteq \operatorname{supp} f_{n} \subseteq \Omega_{n-1}$, where $\Omega_{0}=\Omega$, and $f_{n} \notin 2 n V, f_{n}$ taking the constant value $x_{n}$ in $\Omega_{n}$, for each $n \in \mathrm{N}$. Setting $g_{n}=f_{n}-\chi_{\Omega_{n}} x_{n}$ for each $n$, then according to Lemma 4.2 there is some $k \in \mathrm{N}$ such that $\chi_{\Omega_{n}} x_{n} \in n V$ for each $n \geq k$. Hence $g_{n} \notin n V$ for each $n \geq k$.

If $\left\{A_{n m}: m \in \mathrm{N}\right\}$ is the sequence of the pairwise disjoint elements of $\Sigma$ defined by the non-null values of $g_{n}$, i.e., for each positive integer $m$ there is some $x_{m} \neq 0$ with $A_{n m}=g_{n}^{-1}\left(x_{m}\right)$, then, due to the fact that the $g_{n}$ have disjoint supports, $\left\{A_{n m}: n, m \in \mathrm{N}\right\}$ is a countable family of pairwise disjoints elements of $\Sigma$. So there is some $\pi \in \Pi$ such that $g_{n} \in K(\pi, X)$ for each $n \in \mathbf{N}$ and hence there exists some positive integer $k$ such that $g_{k} \in k V$, a contradiction.

Lemma 4.4. Let $\mathscr{A}$ be an algebra of subsets of $\mathrm{N}$ containing the finite sets. Then $B(\mathscr{A}, X)$ is an ultrabornological space if and only if $X$ is an ultrabornological space.

Proof. Let $V$ be an absolutely convex subset of $B(\mathscr{A}, X)$ which meets each Banach space $L$ contained in $B(\mathscr{A}, X)$ in a neighbourhood of the origin in $L$. A simple modification of the argument of the proof of Lemma 2.4 yields some finite set $J \subseteq \mathrm{N}$ such that $V$ absorbs the closed unit ball of $B(\mathrm{~N} \backslash J, X)$. As $B(J, X)$ is isomorphic to $X^{J}$ since $\Sigma$ contains the singletons, and due to the fact that $X^{J}$ is ultrabornological, then $V$ absorbs the unit ball of $B(J, X)$ and we are done. 
COROllary 4.6. If $X$ is ultrabornological and $\Sigma$ is a $\sigma$-algebra, then the space $K(\Sigma, X)$ is ultrabornological.

Proof. This is an obvious consequence of the two previous results since it is plain that each subspace $K(\pi, X)$ is isomorphic either to $X^{n}$, for some $n \in \mathrm{N}$, or to some space $B(\mathscr{A}, X), \mathscr{A}$ being the $\sigma$-algebra of all subsets of $\mathrm{N}$.

Corollary 4.6. If $X$ is UBL and $\Sigma$ is a $\sigma$-algebra, then $K(\Sigma, X)$ is an inductive limit of UBL spaces.

Proof. Now $X^{n}$ is a UBL space, since it is the product of UBL spaces, and $B(\mathscr{A}, X)$ is also a UBL space according to Proposition 2.5.

\section{The spaces $\operatorname{St}(I, X)$ and $\operatorname{Reg}(I, X)$}

We denote by $\operatorname{Reg}(I, X)$ the linear space of regulated functions, i.e. the linear space of all those $X$-valued bounded functions defined in $I=(0,1]$ which are the uniform limit of a sequence of step functions. By St $(I, X)$ we will denote the linear space formed by the step functions, and assume both spaces provided with the supremum-norm. Identifying $\mathrm{St}(I, X)$ with a subspace of $B(\Sigma, X), \Sigma$ being the algebra of all finite unions of right-semiclosed intervals contained in $I$, we have that $\operatorname{St}(I, X)=\ell_{0}^{\infty}(\Sigma, X)$. Hence $\operatorname{Reg}(I, X)$ coincides with $B(\Sigma, X)$.

Proposition 5.1 St $(I, X)$ is never a barrelled space.

Proof. If $X$ has dimension one let us write $\operatorname{St}(I)$ instead of St $(I, X)$. It suffices to show that $\mathrm{St}(I)$ is not barrelled, since given some fixed $x^{*} \in X^{*}$ with $\left\|x^{*}\right\|=1$, the mapping $T: \mathrm{St}(I, X) \rightarrow \mathrm{St}(I)$ defined by $T f=x^{*} f$ for each $f \in \operatorname{St}(I, X)$ is a quotient map, for $T$ is continuous, and if $x \in X$ is such that $x^{*} x=1$ and $B_{\mathrm{St}(\mathrm{I})}$ and $B_{\mathrm{St}(\mathrm{I}, \mathrm{X})}$ denote the closed unit ball of $\mathrm{St}(I)$ and $\mathrm{St}(I, X)$ respectively, then $\|x\|^{-1} \cdot B_{\mathrm{St}(I)} \subseteq T\left(B_{\mathrm{St}(I, X)}\right)$.

Consider the sequence $\left(f_{n}\right)$ of scalar functions on $I$ defined by $f_{1}(t)=$ $2 \forall t \in(0,1 / 2], f_{1}(t)=-2 \forall t \in(1 / 2,1], f_{2}(t)=8 \forall t \in(1 / 2,3 / 4], f_{2}(t)=-8 \forall t \in(3 / 4,1]$ and $f_{2}(t)=0$ elsewhere, $f_{3}(t)=32 \forall t \in(3 / 4,7 / 8], f_{3}(t)=-32 \forall t \in(7 / 8,1]$ and $f_{3}(t)=0$ in any other point of $I$, and so on. If $\mathscr{A}$ denotes the family of all semiclosed (on the right) intervals contained in $I$ and $J \in \mathscr{A}$, two cases are in order. If $\sup J<1$ there is an $m \in \mathrm{N}$ such that $\operatorname{supp} f_{n} \cap J=\emptyset$ for each $n \geq m$ and consequently $\int_{J} f_{n}(t) d t=0$ for each $n \geq m$. On the other hand, if $\sup J=1$ there is some $m \in \mathrm{N}$ such that $\operatorname{supp} f_{n} \subseteq J$ for each $n \geq m$. Hence one has again that $\int_{J} f_{n}(t) d t=0$ for each $n \geq m$. Therefore, in any case

$$
\lim _{n \rightarrow \infty} \int_{J} f_{n}(t) d t=0 \forall J \in \mathscr{A} .
$$


Defining $u_{n}$ such that $\left\langle u_{n}, g\right\rangle=\int_{0}^{1} f_{n}(t) g(t) d t$ for each $n \in \mathrm{N}$ and each $g \in \operatorname{St}(I)$ then, as a consequence of what we have just seen, the set $U=\left\{u_{n}: n \in \mathrm{N}\right\}$ is weak* bounded in $\mathrm{St}(I)^{*}$. In fact, $\left\langle u_{n}, \chi_{J}\right\rangle=\int_{J} f_{n}(t) d t$ for each $J \in \mathscr{A}$ and each $n \in \mathrm{N}$. But $U$ is not bounded under the norm of $\operatorname{St}(I)^{*}$, since $\int_{0}^{1 / 2} f_{1}(t) d t=1, \int_{1 / 2}^{3 / 4} f_{2}(t) d t=2, \int_{3 / 4}^{7 / 8} f_{3}(t) d t=4$, et cetera.

TheOrem 5.2. $\operatorname{Reg}(I, X)$ is barrelled if and only if $X$ is barrelled.

Proof. Since $\operatorname{Reg}(I, X)$ coincides with $B(\Sigma, X)$, this is an obvious consequence of Corollary 3.4.

Acknowledgment. The author is indebted to the referee for his valuable comments and suggestions.

\section{REFERENCES}

1 L. Drewnowski, M. Florencio and P.J. Paúl, On the barrelledness of spaces of bounded vector functions, Arch. Math. 63 (1994), 449-458.

2 J.C. Ferrando, J.C., A projective description of the simple scalar function space, Rev. Real Acad. Ciencias Madrid 86 (1992), 231-236.

3 J.C. Ferrando, On the barrelledness of the vector-valued bounded function space, J. Math. Anal. Appl. 184, No. 3 (1994), 437-440.

4 J.C. Ferrando, Unordered Baire-like vector-valued function spaces, Bull. Belg. Math. Soc. 2 (1995), 223-227.

5 J.C. Ferrando, M. López Pellicer, L.M. and Sánchez Ruiz, Metrizable barrelled spaces, Pitman Res. Notes Math. Ser. 332, 1995.

6 J.C. Ferrando, L.M. and Sánchez Ruiz, Strong barrelledness properties in $B(\Sigma, X)$, Bull. Austral. Math. Soc. 52 (1995), 207-214.

7 F.J. Freniche, Barrelledness of the space of vector valued and simple functions, Math. Ann. 267 (1984), 479-486.

8 J. Kakol and W. Roelcke, On the barrelledness of $\ell^{p}$-direct sums of seminormed spaces for $1 \leq p \leq \infty$, Arch. Math., to appear.

9 J. Mendoza, Barrelledness conditions on $S(\Sigma, E)$ and $B(\Sigma, E)$, Math Ann. 261 (1982), 11-22.

10 P. Perez Carreras, and J. Bonet, Barrelled locally convex spaces, Notas de Mat. 131, 1987.

11 S.A. Saxon, Nuclear and product spaces, Baire-like spaces and the strongest locally convex topology, Math. Ann. 197 (1972), 87-106.

12 A.R. Todd, and S.A. Saxon, A property of locally convex Baire spaces, Math. Ann. 206 (1973), 23-34.

13 M. Valdivia, On suprabarrelled spaces, Lecture Notes in Math. 843. Func. Anal., Holomorphy and Approximation Theory, Rio de Janeiro 1978, pp. 572-580, 1981.

14 M. Valdivia, On Baire-hyperplane spaces, Proc. Edinburgh Math. Soc. 22 (1979), 247-255.

15 M. Valdivia, and P. Pérez Carreras, On totally barrelled spaces, Math. Z. 178 (1981), 263269.

DEPARTAMENTO DE MATEMÁTICA APLICADA

UNIVERSIDAD MIGUEL HERNANDEZ DE ELCHE

SPAIN

E-mail address: jc.ferrando@umh.es 